\title{
Estimulación magnética transcraneal
}

\section{Transcranial magnetic stimulation}

\author{
M.A. Ibiricu, G. Morales
}

\section{RESUMEN}

La estimulación magnética transcraneal en un método no invasivo para estimular eléctricamente el córtex cerebral y poder valorar la excitabilidad del córtex motor y del tracto corticoespinal. Puede aplicarse mediante pulsos únicos, pares de estímulos separados por intervalos de tiempo variables sobre la misma región o diferente, o mediante trenes de estímulos a frecuencias variables. Estímulos únicos pueden despolarizar neuronas y producir efectos medibles. Trenes de estímulos pueden modificar la excitabilidad cortical de la zona estimulada y de áreas remotas relacionadas a través de conexiones anatómicas funcionales. La estimulación magnética transcraneal puede dar pistas en la fisiopatología de los circuitos neuronales que subyacen en enfermedades neurológicas y psiquiátricas, pero se necesitan más estudios para establecer el papel de la estimulación magnética transcraneal en la clínica neurológica.

Palabras clave. Estimulación magnética transcraneal.

\begin{abstract}
Transcranial magnetic stimulation is a non-invasive tool for the electrical stimulation of the cerebral cortex, and for evaluation of the excitability of the motor cortex and pathways. Transcranial magnetic stimulation can be applied as single pulses of stimulation, pairs of stimuli separated by variable intervals to the same or different brain areas, or as trains of repetitive stimuli at various frequencies. Single stimuli can depolarise neurons and evoke measurable effects. Series of stimuli (repetitive transcranial magnetic stimulation) can modify the excitability of the cerebral cortex at the stimulated site and also at remote areas along functional anatomical connections. TMS might provide novel insights into the pathophysiology of the neural circuitry underlying neurological and psychiatric disorders, but more work is needed to establish the role of transcranial magnetic stimulation in clinical neurology.
\end{abstract}

Key words. Transcranial magnetic stimulation.

An. Sist. Sanit. Navar. 2009; 32 (Supl. 3): 105-113

Servicio de Neurofisiología Clínica. Hospital Virgen del Camino. Pamplona.

\section{Correspondencia}

$\mathrm{M}^{\mathrm{a}}$ Asunción Ibiricu Yanguas

Servicio de Neurofisiología Clínica

Hospital Virgen del Camino

Irunlarrea, 4

31008 Pamplona

Tfno. 848429474

E-mail: mibiricy@cfnavarra.es 


\section{INTRODUCCIÓN}

El primer método de estimulación eléctrica transcraneal eficaz fue utilizado por Merton y Morton en $1980^{1}$. Mostraron que una corriente eléctrica de alto voltaje sobre el cuero cabelludo era capaz de activar el córtex motor, produciendo una contracción de los músculos contralaterales y que el estímulo en la corteza visual producía fosfenos. Sin embargo, este tipo de estimulación es dolorosa por lo que no se utiliza en la clínica diaria. Tiene importancia teórica y sigue utilizándose en situaciones en las que la información clínica y científica no está disponible con estimulación magnética.

Barkers y col en $1985^{2}$ diseñaron un estimulador electromagnético y por primera vez lograron estimular el córtex motor a través del cuero cabelludo. Este tipo de estimulación es poco dolorosa y no invasiva.
El estado de las investigaciones neurofisiológicas del córtex cerebral, especialmente del córtex motor, hasta hace poco confinado a estudios en animales, ha cambiado drásticamente debido a la estimulación magnética transcraneal (EMT).

\section{ASPECTOS FISIOLÓGICOS}

Cuando el cerebro humano es estimulado transcranealmente, una secuencia compleja de eventos le sigue, con efectos tanto excitatorios como inhibitorios. Estos efectos dependen de la intensidad del estímulo, de la excitabilidad del córtex y de la médula.

El estimulador consiste en un condensador que se descarga a través de una bobina y produce un campo magnético que, a través del cuero cabelludo, induce a su vez un campo eléctrico (Fig 1).

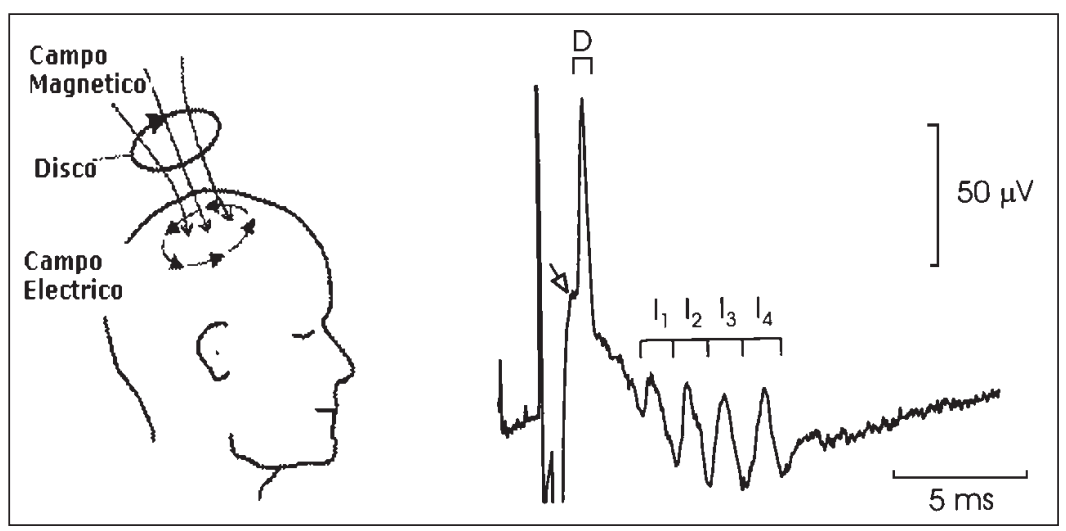

Figura 1. Principio de EMT. La corriente que fluye brevemente en la bobina genera un campo magnético que induce una corriente eléctrica de dirección opuesta, en los tejidos subyacentes. Se evocan múltiples respuestas, la primera u onda directa (D) seguida por varías ondas, denominadas I.

Con una bobina circular y la corriente fluyendo en dirección horaria se excita preferentemente el hemisferio izquierdo. Dando la vuelta a la bobina, de tal forma que la corriente sea en sentido antihorario, se activa preferentemente el hemisferio derecho. Con una bobina en forma de ocho, la zona de intersección de ambos anillos tiene que estar sobre el área motora a estimular.

La EMT evoca múltiples respuestas en las neuronas corticoespinales. La primera o de menor latencia se denomina onda directa o D y las siguientes, ondas indirectas ó I. Las ondas I siguen a la onda D con un intervalo de $1,5 \mathrm{~ms}$ a $2 \mathrm{~ms}$ durante $10 \mathrm{~ms}$. 
Se numeran de acuerdo con su latencia y se denominan como I1, I2, I3 y I4. Hay evidencias de que la primera onda o D se produce por activación directa de las neuronas corticoespinales, mientras que el resto (ondas I) se originan por activación indirecta, a través de interneuronas corticoespinales $^{3}$.
La EMT del córtex motor evoca respuestas EMG en los músculos contralaterales, denominados potenciales evocados motores (PEM) (Fig. 2), que suceden con una latencia breve, compatible con la conducción a través de axones corticoespinales de propagación rápida. Tanto las ondas D como las I contribuyen a la respuesta EMG.

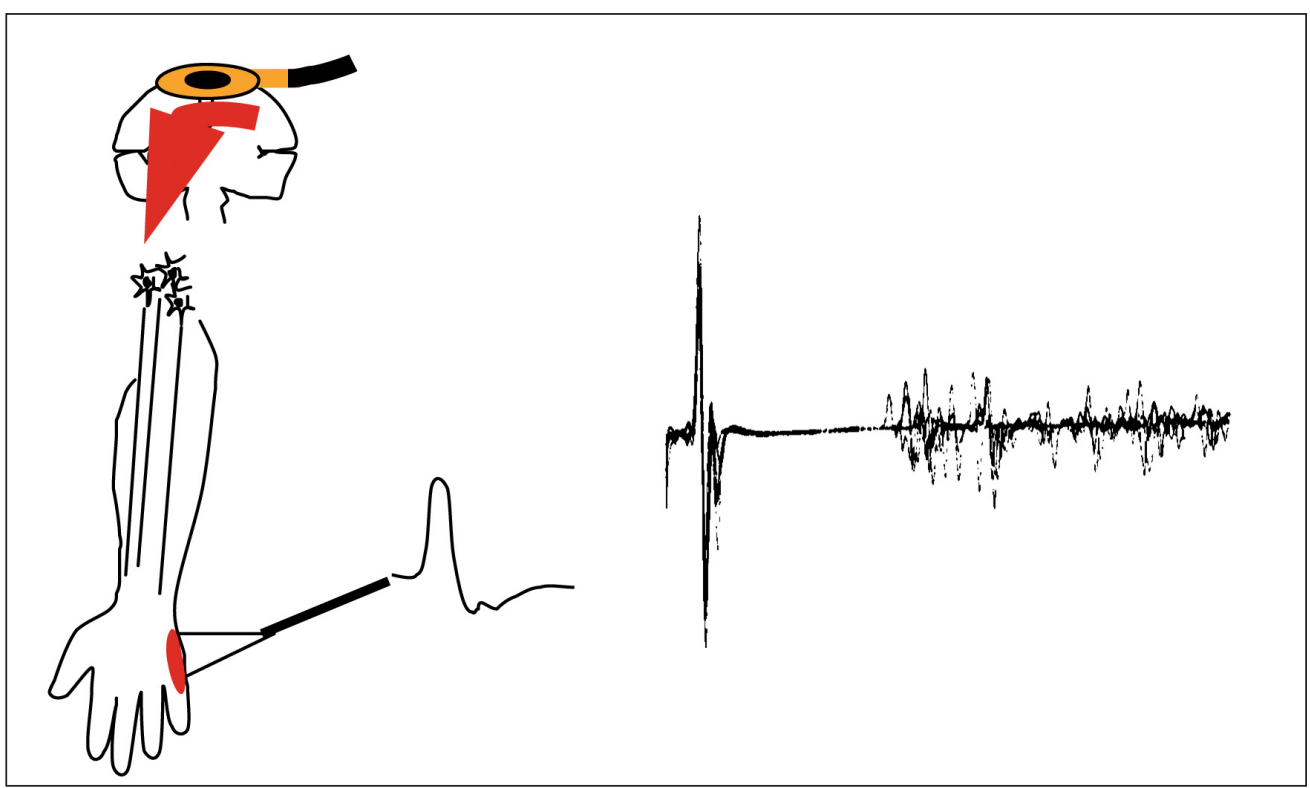

Figura 2. Evocación de respuestas EMG en músculos contralaterales provocadas por la EMT, denominados PEM. Período de silencio cortical.

La contracción voluntaria facilita el PEM, de forma que aumenta su amplitud y disminuye su latencia varios ms. Esto sugiere que cuando el músculo diana es activado voluntariamente, la onda D y la primera I pueden ser suficientes para que se produzca una descarga en las células del asta anterior de la médula, mientras que cuando el músculo está relajado, la onda D y la primera I deben sumarse a las sucesivas I para que se activen las motoneuronas espinales.

\section{VALORACIÓN DEL TRACTO CORTICOESPINAL}

\section{Tiempo de conducción central}

El tiempo de conducción central (TCC) es el tiempo de conducción desde el córtex motor hasta las motoneuronas alfa espinales. Consiste en la diferencia entre el tiempo de conducción desde el córtex hasta el músculo y el tiempo de conducción motor periférico. Para calcular el tiempo motor periférico puede utilizarse la latencia de la onda $\mathrm{F}$, o la estimulación eléctrica o magnética de las raíces nerviosas espinales. Se recomienda medir el TCC mientras el músculo diana esta contraído un 5\%-20\% de la máxima fuerza ${ }^{4}$, ya que el tamaño del PEM se satura con contracciones fuertes.

\section{Tamaño del PEM}

La amplitud del PEM se expresa normalmente como el porcentaje de la amplitud máxima de la onda $\mathrm{M}$, obtenida en el mismo músculo, mediante una estimulación eléc- 
trica supramáxima del nervio correspondiente. De esta forma nos da una medida de la porción de la cantidad de motoneuronas espinales que descargan con la EMT.

\section{VALORACIÓN DE LA EXCITABILIDAD DEL CÓRTEX MOTOR}

\section{Umbral motor}

El umbral motor en reposo (UM) se define como la intensidad mínima (expresada como porcentaje del máximo poder de salida del estimulador) que puede producir un PEM reproducible en un músculo en reposo en el $50 \%$ de 10 a 20 disparos $^{5}$. En los miembros superiores, los PEM recogidos en los músculos intrínsecos de la mano y los obtenidos en los flexores y extensores del antebrazo muestran los valores más bajos de UM. En los miembros inferiores, los UM más bajos se registran en músculo tibial anterior y en el extensor corto de los dedos. Se han atribuido esas diferencias al poder de las proyecciones corticoespinales $^{6}$.

La variabilidad interindividual del UM es alta, lo cual disminuye su aplicación en estudios comparativos de grupos. Sin embargo, las diferencias de UM entre músculos homólogos, derechos e izquierdos (diferencias interhemisféricas) muestran mínimos cambios en los sujetos adultos, por lo que constituye un buen método para detectar alteraciones de la excitabilidad cortical de un hemisferio?

Estudios neurofarmacológicos indican que el UM no se afecta por drogas que modulan el GABA intracortical o la actividad glutamatérgica, mientras que aumenta con drogas que bloquean los canales de membrana voltaje-dependiente ${ }^{8}$. Estas observaciones sugieren que el UM refleja la excitabilidad de las membranas neuronales, la cual depende principalmente de la conductividad de los canales iónicos ${ }^{9}$.

El UM también depende de la excitabilidad de las motoneuronas espinales. El umbral "activo" (el umbral medido durante la contracción voluntaria del músculo diana) es inferior al UM en reposo ${ }^{10}$. Este decremento es el resultado de mecanismos tanto espinales como corticales.

\section{Período de silencio cortical}

Cuando se le pide a un sujeto que mantenga una contracción, la EMT produce una supresión de la actividad electromiográfica después del PEM. Este periodo de "silencio» electromiográfico se denomina periodo de silencio cortical (Fig. 2).

El periodo de silencio (PS) después de un PEM tiene una duración variable en diferentes músculos, en relación con la intensidad del estímulo. Con intensidades de estímulo elevadas es posible que dure más de 200 ms en músculos intrínsecos de la mano. Durante la fase inicial del PS (los primeros 30-50 ms) hay una profunda depresión del reflejo $\mathrm{H}$, demostrando que esa primera parte del PS está producida por mecanismos espinales. Debido a que la amplitud del reflejo $\mathrm{H}$ se recobra antes del final de la pausa EMG, la última parte del PS se considera que tiene un origen supraespinal $^{11}$.

El papel del córtex motor en la génesis del PS se confirma también con la observación de que la duración del PS es mayor después de EMT, que tras estimulación eléctrica transcraneal (EET) ${ }^{12}$. Esto es debido a que la EMT activa predominantemente elementos corticales mientras que la EET activa principalmente axones corticoespinales.

El mecanismo cortical responsable del PS es debido la inhibición de las motoneuronas corticales y la pérdida del impulso excitatorio cortical a las motoneuronas espinales.

Independientemente del mecanismo cortical que subyace en la generación del PS (inhibición de las células corticoespinales o supresión del impulso voluntario motor o ambos), se ha propuesto que están implicados receptores GABA a y GABA b ${ }^{13}$. Sin embargo, la manipulación farmacológica del PS ha mostrado resultados contradictorios, como son, tanto un incremento ${ }^{14}$ como un decremento ${ }^{15}$ de la duración del PS después de dar fármacos GABAérgicos. 


\section{ESTIMULACIÓN MAGNÉTICA DOBLE}

\section{Inhibición y facilitación intracortical}

La excitabilidad cortical puede ser investigada mediante dos estímulos magnéticos en un paradigma de estímulo condicionante (EC)-estímulo test (ET). El efecto modulatorio del EC (primero) en la respuesta evocada por el ET (segundo) depende de la intensidad de los dos estímulos, del intervalo entre ellos y de la presencia o ausencia de contracción muscular voluntaria. Se han propuesto varios paradigmas para estudiar la excitabilidad cortical en individuos adultos y también en pacientes con alteraciones neurológicas.

\section{Inhibición corticocortical}

Un estímulo condicionante sobre el córtex motor, de intensidad tan baja que no puede evocar un PEM, suprime el PEM evocado por un ET supraumbral dado entre 1 y 5 ms después.

La máxima supresión es inducida cuando la intensidad del EC oscila entre el 70 y $90 \%$ del UM en reposo, mientras que intensidades elevadas generan menos inhibición e incluso facilitación.

Dado que el EC no produce supresión del reflejo $\mathrm{H}$ en los músculos flexores del antebrazo, ni en la respuesta EMG evocada en los músculos de la mano por un EET anodal de baja intensidad, se consideró que este fenómeno era de origen cortical; de ahí el término inhibición intracortical (ICI) ${ }^{15}$.

Comparando con los resultados obtenidos en animales de experimentación, Kujirai ${ }^{15}$ propone que esta inhibición es el resultado de la activación de neuronas intracorticales GABAérgicas. Esta hipótesis ha sido confirmada con la manipulación farmacológica. Se pudo mostrar que los fármacos que incrementan la transmisión GABAérgica, aumentando la cantidad de inhibición ${ }^{14}$.

\section{Facilitación intracortical}

Con un paradigma similar al empleado para estudiar la inhibición corticortical, pero con intervalos interestímulo mayo- res, que van desde 6 hasta $20 \mathrm{~ms}$, se ha demostrado una facilitación del $\mathrm{PEM}^{15}$. Esta facilitación sólo está presente cuando el músculo diana está relajado. Durante la contracción, la facilitación se trasforma en una inhibición pequeña, al menos para los intervalos interestímulo que van desde 6 hasta $15 \mathrm{~ms}^{16}$.

Aunque se ha demostrado que la estimulación transcraneal subumbral aumenta la excitabilidad espinal, la facilitación del PEM puede ocurrir a nivel cortical, dado que el EC es muy bajo, aproximadamente $5 \%$ inferior al UM activo ${ }^{17}$. En este caso, el término facilitación intracortical (ICF) parece apropiado ${ }^{18}$.

Sin embargo, debido a que el umbral para este fenómeno es alto, $80 \%$ del UM activo $^{17}$, es obvio que el rango de la intensidad conveniente del estímulo condicionante es bastante estrecho. Por esta razón, es necesaria una determinación precisa del UM, limitando la aplicación clínica de la ICF como una medida de la excitabilidad cortical.

La facilitación intracortical depende de la dirección de la corriente condicionante, mientras que la ICI no. Estos hechos prueban que hay diferentes redes de elementos corticales que son responsables de estos dos fenómenos ${ }^{17}$.

La cantidad de ICF disminuye con fármacos antiglutamatérgicos ${ }^{19,20}$ y por todos los que potencian la actividad GABAérgi$\mathrm{ca}^{14}$.

\section{Aplicaciones diagnósticas y pronósticas de la EMT}

Los hallazgos en estudios con EMT pueden ayudar a localizar el nivel de la lesión a lo largo de los diferentes niveles del sistema motor, distinguiendo entre una lesión predominantemente desmielinizante o axonal predecir el resultado funcional motor tras una lesión.

Las anomalías mostradas por EMT no son específicas de una enfermedad y los resultados deben interpretarse en el contexto de otros datos clínicos ${ }^{21}$ (Tabla 1). 
Tabla 1. Aplicación diagnóstica de la estimulación magnética transcraneal.

\begin{tabular}{lll}
\hline \multicolumn{1}{c}{ TMS Measure } & \multicolumn{1}{c}{ Abnormal Findings } & \multicolumn{1}{c}{ Diseases and Symptoms } \\
\hline CMCT & Long & MS, MND, stroke, secondary parkinsonism, secondary dystonia, brain \\
MEP & injury, SCI, CS & MS, stroke \\
& Dispersed & MS, MND, stroke, brain injury, SCI, CS \\
& Small or absent & Parkinson's disease, dystonia \\
MEP with & Large & MS, MND (with upper motor neuron damage), stroke, secondary \\
triple-stimulation & Central conduction failure & parkinsonism, brain injury, SCI, CS, hydrocephalus \\
technique & & \\
Silent period & Long & MS, stroke, brain injury, SCI, CS, epilepsy, demyelinating neuropathy \\
& Short & MND, Parkinson's disease, dystonia, agenesis of corpus callosum \\
& Absent & SCI, CS \\
Interhemispheric & Long latency & MS, stroke, brain injury (with transcallosal lesion), dysgenesis of corpus \\
conduction & & callosum \\
& Reduced interhemispheric inhibition & MS, MND \\
& Interhemispheric inhibition absent & Stroke (with transcallosal lesion), dysgenesis of corpus callosum \\
Motor cortex & High motor threshold & MS, stroke, agenesis of corpus callosum, brain injury, spinal cord injury, CS \\
excitability & Low motor threshold & MND, epilepsy \\
& Increased intracortical inhibition & Early MND \\
& Decreased intracortical inhibition & Parkinson's disease, SCI, CS, epilepsy \\
& Enlarged cortical representation & Dystonia \\
\hline
\end{tabular}

CMCT, central motor conduction time; CS, cervical spondylosis; $M E P$, motor evoked potential; $M N D$, motor neuron disease; $M S$, multiple sclerosis; SCI, spinal cord injury. Adaptado de Kobayashi M, Pascual-Leone A: Transcranial magnetic stimulation in neurology. Lancet Neurology, 2:145, 2003, with permission.

\section{ESTIMULACIÓN MAGNÉTICA TRANSCREANEAL Y NEUROCIRUGÍA}

La EMT puede utilizarse para la evaluación preoperatoria de áreas específicas del cerebro.

Antes del procedimiento quirúrgico es necesario identificar el hemisferio dominante, localizar las áreas del lenguaje o las áreas motoras. La combinación de EMT y la resonancia magnética funcional pueden ayudar en dichas localizaciones.

La EMT repetitiva de alta frecuencia sobre el hemisferio dominante puede inducir un stop del habla que ayuda a localizar las áreas relacionadas con el lenguaje ${ }^{22}$. Tokimura ${ }^{23}$ describió un método más sencillo para identificar el hemisferio dominante, con un único pulso magnético que medía el aumento de la excitabilidad motora cortical del hemisferio dominante pero no del no dominante durante tareas de lenguaje. La correlación de estos resultados con los del amobarbital intracarotídeo (test de Wada) es alta pero no satisfactoria para un estudio prequirúrgico.
Es importante monitorizar la integridad de la vía motora central durante la cirugía para conseguir mejorar los resultados quirúrgicos. El registro único de los potenciales somatosensoriales no es totalmente satisfactorio ya que un daño en la columna anterior o lateral pueden causar parálisis sin afectar a la columna posterior y por tanto sin alterar los potenciales somatosensoriales.

Los PEM pueden verse afectados por los anestésicos inhalados. Pueden suprimir las ondas indirectas. El desarrollo de nuevos anestésicos y la estimulación repetitiva a alta frecuencia pueden mejorar los registros.

En los procedimientos quirúrgicos en la vecindad del córtex motor, la EMT no ofrece una ventaja real sobre la estimulación eléctrica directa, que puede realizarse más fácilmente en la sala de operaciones; sin embargo, durante la cirugía de tronco del encéfalo y médula o para intervenciones con anestesia espinal, la EMT puede ser más ventajosa debido a que es menos dolorosa y puede ser mas focal que la estimulación eléctrica transcraneal. 


\section{ESTIMULACIÓN MAGNÉTICA TRANSCRANEAL REPETITIVA}

\section{Técnica}

Un tren de pulsos de EMT de la misma intensidad aplicado en una determinada área del cerebro y a una frecuencia que puede variar desde una a veinte estímulos por segundo, se conoce como EMT repetitiva (EMTr). Cuanto mayor es la frecuencia del pulso y la intensidad de la estimulación, mayor es la disrupción de la función cortical durante el tren de estímulo. Sin embargo, después de este efecto inmediato, un tren de estimulación repetitiva puede también inducir un cambio en la excitabilidad cortical. Este efecto puede ir desde inhibición hasta facilitación, dependiendo de las variables de estimulación, principalmente de la frecuencia de estimulación. Frecuencias bajas, del orden de $1 \mathrm{~Hz}$, puede suprimir la excitabilidad del córtex motor, mientras que trenes de frecuencias del rango de $20 \mathrm{~Hz}$ pueden producir un incremento temporal de la excitabilidad cortical ${ }^{24}$.

Varios estudios combinando EMTr y técnicas de neuroimagen funcional han detectado descenso o incremento del flujo sanguíneo cerebral y el metabolismo en el área estimulada después de trenes de estimulación a bajas o altas frecuencias respectivamente ${ }^{25}$.

Los mecanismos de la modulación de la excitabilidad cortical no están claros. La potenciación o depresión a largo tiempo de las sinapsis corticales o mecanismos neuronales relacionados han sido sugeridas como posibles mecanismos para explicar los efectos de la EMTr a altas y bajas frecuencias respectivamente. Estudios con animales sugieren que la modulación de los trasmisores y la inducción genética pueden contribuir a esos efectos modulatorios de la EMTr.

\section{Uso terapéutico}

El tratamiento de la depresión es el efecto más ampliamente estudiado de la aplicación clínica de la EMTr. Se ha visto que puede producir un beneficio en el $40 \%$ de pacientes con medicaciones resistentes a la depresión ${ }^{26}$.

Se han hecho estudios en pacientes con enfermedad de Parkinson en los que EMTr a altas frecuencias mejoraba la función de la mano contralateral.

También en distonías, en las que se sugiere que existe una hiperexcitabilidad del córtex motor o un fallo de la inhibición intracortical, se ha utilizado EMTr a $1 \mathrm{~Hz}$ en el calambre del escribiente.

Varios estudios han utilizado estimulaciones a bajas frecuencias para el tratamiento de epilepsias mal controladas con fármacos y en el mioclonus cortical. En todos estos estudios, sólo en pocos pacientes se han encontrado resultados positivos y los hallazgos son contradictorios.

La recuperación tras un accidente cerebrovascular puede verse influido favorablemente por la EMTr, suprimiendo la plasticidad cortical maladaptada y promoviendo una actividad cortical adecuada para promover la neurorehabilitación. Estas observaciones son prematuras para promoverlas como aplicación terapeútica. Sin embargo, la EMTr de regiones de interés detectadas en imágenes funcionales, puede poner de relieve las propiedades de los cambios plásticos de los circuitos corticales y pueden darnos pistas de las futuras intervenciones clínicas.

\section{CONSIDERACIONES DE SEGURIDAD}

Efectos adversos tras EMT de un solo pulso sobre el córtex motor es muy rara. El efecto potencialmente adverso que han provocado más inquietud hasta ahora ha sido epilepsia, aunque teóricamente el riesgo es remoto. Varios miles de pacientes han sido sometidos a estimulación eléctrica y magnética pero solamente se han descrito aisladas crisis focales.

En contraste, no hay discusión que EMTr a altas frecuencias pueden producir crisis en sujetos normales además de en pacientes con alteraciones neurológicas. La evaluación de los parámetros de estímulo pertinente a la inducción de las crisis 
(intensidad del estímulo, frecuencia, y la duración del tren y la frecuencia) ha llevado a unas específicas recomendaciones y una guía para EMTr27.

Teóricamente, estructuras metálicas implantadas dentro del cráneo (clips de aneurismas) pueden moverse por la fuerza mecánica de la corriente inducida, aunque es muy improbable. Es razonable tener en cuenta ambos, epilepsia y neurocirugía previa como una relativa más que absoluta contraindicación de la estimulación.

Hay que tener precaución con los implantes cocleares. Se ha sugerido que los artefactos acústicos de la bobina del estimulador magnético pueden producir pérdida auditiva y se recomienda el uso de tapones. Esto es importante en niños de corta edad en los que la distancia bobina-oído es muy pequeña.

\section{BIBLIOGRAFÍA}

1. Merton PA, Morton HB. Stimulation of the cerebral cortex in the intact human subject. Nature 1980; 285; En: Basic mechanism of TMS. Terao Y, Ugawa Y. J Clin Neurophysiol 2002; 19: 322-343.

2. Barker AJ, Jalinous R, FreEston IL. Non-invasive stimulation of human motor cortex. Lancet 1985; 11:1106-1107; En: Basic mechanism of TMS. Terao Y, Ugawa Y. J Clin Neurophysiol 2002; 19: 322-343.

3. Patton HD, Amassian VE. Single and multiple unit analysis of the cortical stage of pyramidal tract activation. J Neurophysiol 1954; 17: 345-363; En: Clinical and research methods for evaluating cortical excitability; Abbruzzese G, Trompeto C. J Clin Neurophysiol 2002; 19: 307-321.

4. Rossini PM, Beradelli A, Deuschl G, Hallet M, Maertenss de Noordhout A, Paulus W et al. Aplications of magnetic cortical stimulation. The International Federation of Clinical Neurophysiology. Electroencephalogr Clin Neurophysiol 1999; 52: S171-185.

5. Rossini PM, Barker AT, Berardelli A, Caramia MD, Caruso G, CRacco RQ et al. Noninvasive electrical and magnetic sti- mulation of the brain, spinal cord and roots: basic principles and procedures for routine clinical application. Report of an IFCN committee. Electroencephalogr Clin Neurophysiol 1994; 91: 79-92.

6. Brouwer B, Ashby P. Corticospinal projections to upper and lower limb spinal motoneurons in man. Electroencephalogr Clin Neurophysiol 1990; 76: 509-519.

7. Cicinelli P, Traversa R, Bassi P, Scivoletto G, Rossini PM. Interhemispheric differences of hand muscle representation in human motor cortex. Muscle Nerve 1997; 20: 535-542.

8. Ziemann U, Lonnecker S, Steinhoff BJ, PaULus W. Effects of antiepileptic drugs on motor cortex exitability in humans: a transcranial magnetic stimulation study. Ann Neurol 1996; 40: 367-378.

9. Ziemann U, Steinhoff BJ, Tergau F, Paulus W. Transcranial magnetic stimulation: its current role in epilepsy research. Epilepsy Res 1998; 30: 11-30.

10. Hess CW, Mills KR, MurRay NMF. Responses in small hand muscles from magnetic stimulation of the human brain. J Physiol 1987; 338: 397-419.

11. Ziemann U, Netz J, Szelenyi A, Homberg V. Spinal and supraspinal mechanisms contribute to the silent period in the contracting soleus muscle after transcranial magnetic stimulation of human motor cortex. Neurosci Lett 1993; 156: 167-171.

12. Inghilleri M, Berardelli A, Cruccu G, MaNFREDI M. Silent period evoked by transcranial stimulation of the human cortex and cervicomedullary junction. J Physiol 1993; 466: 521-534.

13. Hallet M. Transcranial magnetic stimulation. Negative effects. Adv Neurol 1995; 509: 607-618.

14. Ziemann U, Lönnecker S, Steinhoff BJ, PaULus W. The effect of lorazepam on the motor cortical excitability in man. Exp Brain Res 1996; 109: 127-135.

15. Kujirai T, Caramia MD, Rothwell J. Corticocortical inhibition in human motor cortex. J Physiol 1993; 471: 501-519. 
16. Ridding MC, Taylor JL, Rothwell J. The effect of voluntary contraction on cortico-cortical inhibition in human motor cortex. J Physiol 1995; 487: 541-548.

17. Ziemann U, Rothwell J, Ridding MC. Interaction between intracortical inhibition and facilitation in human motor cortex. J Physiol 1996c; 496: 873-881.

18. Chen R, Tam A, Bütefisch C, Corwell B, Ziemann U, Rothwell J, Cohen LG. Intracortical inhibition and facilitation in different representations of the human motor cortex. J Neurophysiol 1998; 80: 2870-2881.

19. Liepert J, Schwenkreis P, Tegenthoff M, MaLIN JP. The glutamate antagonis riluzole suppresses intracortical facilitation. J Neural Transm 1997; 104: 1207-1214.

20. Ziemann U, Chen R, Cohen LG. Dextromethorphan decreases the excitability of the human motor cortex. Neurology 1998a; 51: 1320-1324.

21. Kobayashi M, Pascual-Leone A. Transcranial magnetic stimulation in neurology. Lancet Neurology 2003; 2: 145-156.

22. Pascual-Leone A, Gates JR, Dhuna A. Induction of speech arrest and counting errors with rapid-rate transcranial magnetic stimulation. Neurology 1991; 41: 697-702.

23. Tokimura H, Tokimura Y, Oliviero A, AsakuRA T, Rothwell JC. Speech induced changes in corticospinal excitability. Ann Neurol 1996; 40: 628-634.

24. Berardelli A, InGHilLeri M, Rothwell JC. Facilitation of muscle evoked responses after repetitive cortical stimulation in man. Exp Brain Res 1998; 122: 79-84.

25. Pascual-Leone A, Tormos JM, Keenan J, TARAZONA F. Study and modulation of human cortical excitability with transcranial magnetic stimulation. J Clin Neurophysiol 1998; 15: 333-343.

26. WasSermann EM, LISAnBy Sh. Therapeutic application of repetitive transcranial magnetic stimulation; a review. Clin Neurophysiol 2001; 112: 1367-1377.

27. Wassermann EM. Safety and side effects of transcranial magnetic stimulation and repetitive transcranial magnetic stimulation. p. 39. En: Pascual-Leone A, Davey NJ, Rothwell J et al (eds): Handbook of Transcranial Magnetic Simulation. Arnold, London, 2002. 